\title{
Connecting Compton and Gravitational Compton Scattering
}

\author{
Barry R. Holstein ${ }^{1}$ \\ ${ }^{1}$ Department of Physics-LGRT, University of Massachusetts, Amherst, MA 01003, USA
}

\begin{abstract}
The study of Compton scattering- $S+\gamma \rightarrow S+\gamma$-at MAMI and elsewhere has led to a relatively successful understanding of proton structure via its polarizabilities. The recent observation of gravitational radiation observed by LIGO has raised the need for a parallel understanding of gravitational Compton scattering- $S+g \rightarrow S+g$-and we show here how it can be obtained from ordinary Compton scattering by use of the double copy theorem.
\end{abstract}

\section{Introduction}

The recent observation at LIGO of gravitational radiation has verified the existence of gravitons and has emphasized the importance of studying processes involving their interactions [1]. In the case of the electromagnetic interaction, the Compton scattering process- $S+\gamma \rightarrow S+\gamma$-has been utilized for many years as a probe of particle structure by measuring the response of a scattering system to external electromagnetic fields, which are characterized by so-called polarizabilities [2]. The use of causality allows another type of probe by the use of various sum rules. An example of this kind is the well-known GDH (GerasimovDrell-Hearn) sum rule, which relates an integral over experimental photoabsorption cross-sections to the anomalous magnetic moment of the target [3],[4]. It is of interest then to study the related process of gravitational Compton scattering- $S+g \rightarrow S+g$. In this case the evaluation of such amplitudes is simplified enormously by use of the double copy theorem, which asserts that such gravitational Compton amplitudes can be expressed in terms of the product of simple Compton scattering amplitudes and an elementary kinematic factor [5],[6]. In the case of the gravitational interaction, angular momentum conservation demands that there can exist no anomalous gravitomagnetic moment and it is then interesting to ask if there is a corresponding gravitational GDH sum rule.

It is the purpose of this note to study such issues. In section 2 then, we review the subject of Compton scattering and its connection with polarizabilities. We show how such quantities have been calculated by use of chiral perturbation theory and confront the theoretical and experimental values. In section 3 we examine the parallel gravitational Compton scattering reaction and show how factorization allows an enormously simplified evaluation of such amplitudes. The validity of sum rules is also studied. In section 4 we show how the gravitational Compton amplitudes can be used together with unitarity to provide an elementary evaluation of higher order gravitational scatter- ing and our results are summarized in a brief concluding section.

\section{Compton Scattering}

The Compton scattering process has long been studied both experimentally and theoretically [7]. On the theoretical side Low's theorem [8] asserts that the Compton scattering amplitude is given up to $O\left(\omega^{2}\right)$ by the simple Born amplitude plus a possible anomalous magnetic moment term [9] where here the Born amplitude arises from the sum of lowest order contributions from the three diagrams shown in Figure 1.

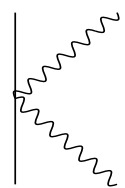

a)

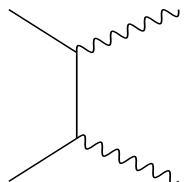

b)

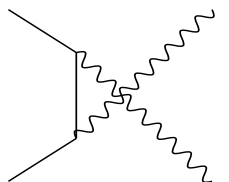

c)
Figure 1. Shown are the a) seagull, b) Born, and c) Cross-Born diagrams contributing to Compton scattering. Here the solid lines represent massive scalars while the wiggly lines are photons.

In the case of a spinless particle, the Compton amplitude is found from the minimal gauged Klein-Gordon Lagrangian

$$
\mathcal{L}(S=0)=\left(D_{\mu} \phi\right)^{\dagger} D^{\mu} \phi-m^{2} \phi^{2}-\frac{1}{4} F_{\mu \nu} F^{\mu v}
$$

where $D_{\mu}=\partial_{\mu}+i e A_{\mu}$ is the covariant derivative and $F_{\mu \nu}=\partial_{\mu} A_{\nu}-\partial_{\nu} A_{\mu}$ is the electromagnetic field tensor. The contributions from the three (individually non-gauge- 
invariant) diagrams are

$$
\begin{aligned}
\operatorname{Amp}_{C}^{1 a}(S=0) & =-2 e^{2} \epsilon_{f}^{*} \cdot \epsilon_{i} \\
\operatorname{Amp}_{C}^{1 b}(S=0) & =\frac{2 e^{2} \epsilon_{f}^{*} \cdot p_{f} \epsilon_{i} \cdot p_{i}}{p_{i} \cdot k_{i}} \\
\operatorname{Amp}_{C}^{1 c}(S=0) & =-\frac{2 e^{2} \epsilon_{f}^{*} \cdot p_{i} \epsilon_{i} \cdot p_{f}}{p_{i} \cdot k_{f}}
\end{aligned}
$$

and the resultant Born amplitude

$$
\begin{gathered}
\operatorname{Amp}_{C}^{B o r n}(S=0) \sum_{i=a}^{c}=\operatorname{Amp}_{C}^{1 i}(S=0) \\
=2 e^{2}\left[\frac{\epsilon_{f}^{*} \cdot p_{f} \epsilon_{i} \cdot p_{i}}{p_{i} \cdot k_{i}}-\frac{\epsilon_{f}^{*} \cdot p_{i} \epsilon_{i} \cdot p_{f}}{p_{i} \cdot k_{f}}-\epsilon_{f}^{*} \cdot \epsilon_{i}\right]
\end{gathered}
$$

is verified to be gauge-invariant. Low's theorem in this case reads [9]

$$
\operatorname{Amp}_{C}^{t o t}(S=0)=\operatorname{Amp}_{C}^{B o r n}(S=0)+\operatorname{Amp}_{C}^{N B}(S=0)
$$

where $\operatorname{Amp}_{C}^{N B}(S=0)=O\left(\omega^{2}\right)$ and possible higher order corrections can be found from an effective Lagrangian

$$
\mathcal{L}_{e f f}=C_{1} F_{\mu \nu} F^{\mu v} \phi^{\dagger} \phi+C_{2} F_{\mu \lambda} F^{\lambda}{ }_{\nu} \partial^{\mu} \phi^{\dagger} \partial^{v} \phi+\ldots
$$

where the ellipsis indicates higher derivative contributions. This result is conventionally written as

$$
H_{e f f}=\frac{(\boldsymbol{p}-e \boldsymbol{A})^{2}}{2 m}-\frac{1}{2} 4 \pi \alpha_{E} \boldsymbol{E}^{2}-\frac{1}{2} 4 \pi \beta_{M} \boldsymbol{B}^{2}+\ldots
$$

where $\alpha_{E}, \beta_{M}$ are the electric, magnetic polarizabilities and measure the size of the induced electric, magnetic dipole moments when the system is placed in an external electric or magnetic field via [2]

$$
\boldsymbol{d}_{E}=4 \pi \alpha_{E} \boldsymbol{E} \text { and } \boldsymbol{d}_{M}=4 \pi \beta_{M} \boldsymbol{B}
$$

Since Eq. (6) implies a Compton scattering cross section

$$
\begin{aligned}
\frac{d \sigma_{C}(S=0)}{d \Omega} & =\frac{\alpha^{2}}{2 m^{2}} \frac{\omega_{f}^{2}}{\omega_{i}^{2}}\left\{1+\cos ^{2} \theta\right. \\
& -\frac{m \omega_{f} \omega_{i}}{\alpha}\left[\left(\alpha_{E}+\beta_{M}\right)(1+\cos \theta)^{2}\right. \\
& \left.\left.+\left(\alpha_{E}-\beta_{M}\right)(1-\cos \theta)^{2}\right]+\ldots\right\}(8)
\end{aligned}
$$

where $\alpha=e^{2} / 4 \pi$ is the fine structure constant, we see that the polarizabilities can be measured via Compton scattering. In a series of measurements over the years we now have reliable and precise values for the proton poarizabilities, as listed by the Particle Data Group [10]

$$
\alpha_{E}^{p}=(11.2 \pm 0.4) \times 10^{-4} \mathrm{fm}^{3}
$$

and

$$
\beta_{M}^{p}=(2.5 \pm 0.4) \times 10^{-4} \mathrm{fm}^{3}
$$

and these numbers contain useful information about proton structure. Thus, since the hydrogen atom electric polarizability is of order the atomic volume, while the proton electric polarizabiity is of order $10^{-3}$ of the proton volume [11], we learn that the proton is a much stiffer object than the atom. Likewise, since the $\Delta$ pole makes a strongly paramagnetic contribution to $\beta_{M}[12]-\beta_{M}^{\Delta-\text { pole }} \sim$ $10 \times 10^{-4} \mathrm{fm}^{3}$ - the measured value given in Eq. (10) indicates a significant diamagnetic contribution, which may arise from the pion cloud.

Causality can be used to write fixed-angle dispersion relations [13]. Thus in the forward direction, we have

$$
\operatorname{Amp}_{C}^{t o t}(S=0) \stackrel{\theta \rightarrow 0}{\longrightarrow} \boldsymbol{\epsilon}_{f}^{*} \cdot \boldsymbol{\epsilon}_{i} f(\omega)
$$

with

$$
f(\omega)=f(-\omega)=-\frac{e^{2}}{4 \pi m}+\left(\alpha_{E}+\beta_{M}\right) \omega^{2}+\ldots
$$

The amplitude $f(\omega)$ obeys a once-subtracted dispersion relation

$$
f(\omega)=-\frac{e^{2}}{4 \pi m}+\frac{\omega^{2}}{2 \pi^{2}} \int_{0}^{\infty} \frac{d \omega^{\prime} \sigma_{t o t}\left(\omega^{\prime}\right)}{\omega^{\prime 2}-\omega^{2}}
$$

where $\sigma_{t o t}\left(\omega^{\prime}\right)$ is the photoabsorption cross section and, expanding both sides of Eq. (13) in $\omega^{2}$, we find the Baldin sum rule [14]

$$
\alpha_{E}+\beta_{M}=\frac{1}{2 \pi^{2}} \int_{0}^{\infty} \frac{d \omega^{\prime} \sigma_{t o t}\left(\omega^{\prime}\right)}{\omega^{\prime 2}}
$$

In the case of spin- $\frac{1}{2}$, the Born term becomes

$$
\begin{aligned}
& \operatorname{Amp}_{C}^{\text {Born }}\left(S=\frac{1}{2}\right)=e^{2} \bar{u}\left(p_{f}\right) \\
\times & {\left[\frac{\left(\xi_{f}^{*}+\frac{i \kappa}{2 m} \sigma_{\mu \nu} \epsilon_{f}^{* \mu} k_{f}^{v}\right)\left(p_{i}+\not k_{i}+m\right)\left(\xi_{i}-\frac{i \kappa}{2 m} \sigma_{\mu \nu} \epsilon_{i}^{\mu} k_{i}^{v}\right)}{2 p_{i} \cdot k_{i}}\right.} \\
- & \left.\frac{\left(\xi_{i}-\frac{i \kappa}{2 m} \sigma_{\mu \nu} \epsilon_{i}^{\mu} k_{i}^{v}\right)\left(p_{f}-k_{i}+m\right)\left(\xi_{f}^{*}+\frac{i \kappa}{2 m} \sigma_{\mu \nu} \epsilon_{f}^{* \mu} k_{f}^{v}\right)}{2 p_{i} \cdot k_{f}}\right] \\
\times & u\left(p_{i}\right)
\end{aligned}
$$

where $\kappa$ is the anomalous magnetic dipole moment. Low's theorem, as before, reads

$$
\operatorname{Amp}_{C}^{t o t}\left(S=\frac{1}{2}\right)=\operatorname{Amp}_{C}^{B o r n}\left(S=\frac{1}{2}\right)+\operatorname{Amp}_{C}^{N B}\left(S=\frac{1}{2}\right)
$$

with $\operatorname{Amp}_{C}^{N B}=O\left(\omega^{2}\right)$, but now the forward scattering amplitude has the form

$\operatorname{Amp}_{C}^{t o t}\left(S=\frac{1}{2}\right) \stackrel{\theta \rightarrow 0}{\longrightarrow} \chi_{f}^{\dagger} \chi_{i} \boldsymbol{\epsilon}_{f}^{*} \cdot \boldsymbol{\epsilon}_{i} f(\omega)+i \chi_{f}^{\dagger} \boldsymbol{\sigma} \chi_{i} \cdot \boldsymbol{\epsilon}_{f}^{*} \times \boldsymbol{\epsilon}_{i} g(\omega)$

with

$$
\begin{gathered}
f(\omega)=f(-\omega)=-\frac{e^{2}}{4 \pi m}+O\left(\omega^{2}\right) \\
\text { and } \\
g(\omega)=-g(-\omega)=\frac{e^{2} \kappa^{2} \omega}{2 \pi m}+O\left(\omega^{3}\right)
\end{gathered}
$$

Causality implies that we still have the Baldin sum rule, which now reads

$$
\alpha_{E}+\beta_{M}=\frac{1}{2 \pi^{2}} \int_{0}^{\infty} \frac{d \omega^{\prime}\left(\sigma_{\frac{3}{2}}\left(\omega^{\prime}\right)+\omega_{\frac{1}{2}}\left(\omega^{\prime}\right)\right)}{\omega^{\prime 2}}
$$


where $\sigma_{\frac{3}{2}}\left(\omega^{\prime}\right), \sigma_{\frac{1}{2}}\left(\omega^{\prime}\right)$ are the photoabsorption cross sections for parallel, antiparallel photon and taget helicities, and we also have a sum rule for $g(\omega)$ which, since it involves the spin-flip amplitude, is unsubtracted

$$
\frac{\pi^{2} e^{2} \kappa^{2}}{2 m^{2}}=\int_{0}^{\infty} \frac{d \omega^{\prime}\left(\sigma_{\frac{3}{2}}\left(\omega^{\prime}\right)-\sigma_{\frac{1}{2}}\left(\omega^{\prime}\right)\right)}{\omega^{\prime}}
$$

Eq. (21) is the GDH (Gerasimov-Drell-Hearn) sum rule [3],[4] and is well-satisfied in the case of the proton [7]

$$
L H S=205 \mu \mathrm{b} \quad \text { vs. } \quad R H S=212 \pm 15 \mu \mathrm{b}
$$

In addition to the leading order polarizabilities $\alpha_{E}, \beta_{M}$, one can probe for additional structure by defining higher order quantities [15]

$$
\begin{aligned}
& H_{e f f}=\frac{(\boldsymbol{p}-e \boldsymbol{A})^{2}}{2 m}-\frac{1}{2} 4 \pi \alpha \boldsymbol{E}^{2}-\frac{1}{2} 4 \pi \beta_{M} \boldsymbol{B}^{2} \\
- & \frac{1}{2} 4 \pi\left(\gamma _ { E 1 E 1 } \boldsymbol { \sigma } \cdot \left(\boldsymbol{E} \times \dot{\boldsymbol{E}}+\gamma_{M 1 M 1} \boldsymbol{\sigma} \cdot(\boldsymbol{B} \times \dot{\boldsymbol{B}}\right.\right. \\
- & \left.2 \gamma_{M 1 E 2} \sigma_{i} B_{j} E_{i j}+2 \gamma_{E 1 M 2} \sigma_{i} E_{j} B_{j k}\right) \\
- & \frac{1}{2} 4 \pi\left(\alpha_{E 1 \nu} \boldsymbol{E}^{2}+\beta_{M 1 v} \dot{\boldsymbol{B}}^{2}\right) \\
- & \frac{1}{12} 4 \pi\left(\alpha_{E 2} E_{i j}^{2}+\beta_{M 2} B_{i j}^{2}\right)+\ldots
\end{aligned}
$$

where $E_{i j}=\frac{1}{2}\left(\nabla_{i} E_{j}+\nabla_{j} E_{i}\right)$ and $B_{i j}=\frac{1}{2}\left(\nabla_{i} B_{J}+\nabla_{j} B_{i}\right)$ are field gradients. These definitions lead to additional sum rules

$$
\begin{gathered}
\alpha_{E 1 v}+\beta_{M 1 v}+\frac{1}{12}\left(\alpha_{E 2}+\beta_{M 2)}\right. \\
=\frac{1}{2 \pi^{2}} \int_{0}^{\infty} \frac{d \omega^{\prime}\left(\sigma_{\frac{3}{2}}\left(\omega^{\prime}\right)+\sigma_{\frac{1}{2}}\left(\omega^{\prime}\right)\right)}{\omega^{\prime 4}} \\
\gamma_{E 1 E 1}+\gamma_{M 1 M 1}+\gamma_{E 2 M 1}+\gamma_{M 2 E 1} \\
=\frac{1}{4 \pi^{2}} \int_{0}^{\infty} \frac{d \omega^{\prime}\left(\sigma_{\frac{3}{2}}\left(\omega^{\prime}\right)-\sigma_{\frac{1}{2}}\left(\omega^{\prime}\right)\right)}{\omega^{\prime 3}}
\end{gathered}
$$

and

$$
\begin{aligned}
& \gamma_{E 1 E 1 v}+\gamma_{M 1 M 1 v}+\gamma_{M 2 E 1 v}+\gamma_{M 2 E 1 v} \\
+ & \gamma_{E 2 E 2}+\gamma_{M 2 M 2}+\frac{8}{5} \gamma_{M 3 E 2}+\frac{8}{5} \gamma_{E 3 M 2} \\
= & \frac{1}{4 \pi^{2}} \int_{0}^{\infty} \frac{d \omega^{\prime}\left(\sigma_{\frac{3}{2}}\left(\omega^{\prime}\right)-\sigma_{\frac{1}{2}}\left(\omega^{\prime}\right)\right)}{\omega^{5}}
\end{aligned}
$$

On the theoretical side, there have been various theoretical approaches used to predict polarizabilities, but recently much work has been done using chiral perturbation theory [16], using both the heavy baryon [17] and covariant baryon versions [18]. In the case of $\alpha_{E}, \beta_{M}$ we have the results shown in Table 1 . The values of the spin polarizabilities recently measured by the A 2 collaboration at MAMI are compared with theoretical predictions in Table 2. Finally, some calculated values of higher order polarizabilities are shown in Table 3 The rough general agreement here between the experimental and theoretical numbers for the polarizabilities suggests the important role played by the pion cloud.

\begin{tabular}{cccc}
\hline & & & \\
& $\mathrm{HB} \chi \mathrm{pt}$ & $\mathrm{B} \chi \mathrm{pt}$ & $\mathrm{PDG}[10]$ \\
\hline & & & \\
$\alpha_{E}$ & $10.7 \pm 0.5$ & $10.6 \pm 0.5$ & $11.2 \pm 0.4$ \\
$\beta_{M}$ & $3.2 \mp 0.5$ & $3.2 \mp 0.5$ & $2.5 \mp 0.4$ \\
& & & \\
\hline
\end{tabular}

Table 1. Shown are theoretical and experimental values of the electric and magnetic polarizabilities compared with the values quoted by the Particle Data Group. All numbers are in units of $10^{-4} \mathrm{fm}^{3}$.

\begin{tabular}{ccccc}
\hline & & & & \\
& DR [19] & $\mathrm{HB} \chi \mathrm{pt}$ & $\mathrm{B} \chi \mathrm{pt}$ & Expt. [20] \\
\hline$\gamma_{E 1 E 1}$ & -5.6 & $-1.1 \pm 1.8$ & $-3.3 \pm 0.8$ & $-3.5 \pm 1.2$ \\
$\gamma_{M 1 M 1}$ & 3.8 & $2.2 \pm 0.6$ & $2.9 \pm 1.5$ & $3.2 \pm 0.9$ \\
$\gamma_{E 1 M 2}$ & -0.7 & $-0.4 \pm 0.4$ & $0.2 \pm 0.2$ & $-0.7 \pm 1.2$ \\
$\gamma_{E 2 M 1}$ & 2.9 & $1.9 \pm 0.4$ & $1.1 \pm 0.4$ & $2.0 \pm 0.3$ \\
& & & & \\
\hline
\end{tabular}

Table 2. Shown are theoretical and experimental values of the spin polarizabilities. All numbers are in units of $10^{-4} \mathrm{fm}^{4}$.

\begin{tabular}{cc}
\hline & $\mathrm{HB} \chi \mathrm{pt}(\mathrm{NNLO})$ \\
\hline$\alpha_{E 2}$ & $17.3 \pm 3.9$ \\
$\beta_{M 2}$ & $-15.5 \pm 3.5$ \\
$\alpha_{E 1 v}$ & $-1.3 \pm 0.4$ \\
$\beta_{M 1 v}$ & $7.1 \pm 2.5$ \\
\hline
\end{tabular}

Table 3. Shown are theoretical values of certain higher order electric and magnetic polarizabilities. All numbers are in units of $10^{-4} \mathrm{fm}^{5}$.

\section{Gravitational Compton Scattering}

When considering gravitational Compton scattering, Low's theorem can again be applied [9] but now any corrections to the simple Born amplitudes must be of order $O\left(\omega^{4}\right)$, where here the Born amplitude arises from the sum of the four diagrams shown in Figure 2.

The transition from electromagnetic to gravitational interactions is at lowest order given by the substitution [21]

$$
\mathcal{L}_{e m}=-e j_{\mu} A^{\mu} \quad \longrightarrow \quad \mathcal{L}_{g r a v}=-\frac{\kappa}{2} T_{\mu \nu} h^{\mu \nu}
$$

Here, in the electromagnetic case, $j_{\mu}=\frac{1}{e} \frac{\delta \mathcal{L}}{\delta A^{\mu}}$ is the electromagnetic current, $\alpha=e^{2} / 4 \pi$ is the fine structure constant, and $A^{\mu}$ is the vector potential, while for the gravitational interaction $T_{\mu \nu}=-\frac{2}{\sqrt{-\operatorname{det} g}} \frac{\delta \mathcal{L}_{\text {mat }}}{\delta g_{\mu \nu}}$ is the energymomentum tensor, $G=\kappa^{2} / 32 \pi$ is the Cavendish constant, and $g_{\mu \nu}=\eta_{\mu \nu}+h_{\mu \nu}$ is the metric tensor. Writing the spin- 0 

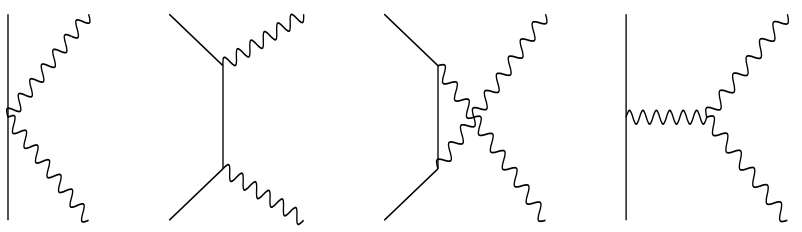

a) c)

d)
Figure 2. Shown are the a) seagull, b) Born, c) Cross-Born, and d) graviton pole diagrams contributing the gravitational Compton scattering. Here the solid lines represent massive spinless particles while the wiggly lines are gravitons.

matter Lagrangian as

$$
\mathcal{L}_{m a t}=g_{\mu \nu}\left(D^{\mu} \phi\right)^{\dagger} D^{v} \phi-m^{2} \phi^{\dagger} \phi
$$

we find the electromagnetic current

$$
j_{\mu}=\phi^{\dagger} \partial_{\mu} \phi-(\partial \phi)^{\dagger} \phi
$$

and energy-momentum tensor

$$
T_{\mu \nu}=\left(\partial_{\mu} \phi\right)^{\dagger} \partial_{\nu} \phi+\left(\partial_{\nu} \phi\right)^{\dagger} \partial_{\mu} \phi-\frac{1}{2} g_{\mu \nu} \mathcal{L}
$$

We have then the general matrix element forms [21]

$$
\left\langle p_{f}\left|j_{\mu}(x)\right| p_{i}\right\rangle=e^{-i q \cdot x} G\left(q^{2}\right) 2 P_{\mu}
$$

and

$$
\begin{aligned}
& \left\langle p_{f}\left|T_{\mu \nu}(x)\right| p_{i}\right\rangle=e^{-i q \cdot x}\left[2 P_{\mu} P_{\nu} F_{1}\left(q^{2}\right)\right. \\
+\quad & \left.\left(q_{\mu} q_{\nu}-\eta_{\mu \nu} q^{2}\right) F_{2}\left(q^{2}\right)\right]
\end{aligned}
$$

where $q=p_{i}-p_{f}$ is the momentum transfer and $P_{\mu}=$ $\frac{1}{2}\left(p_{f}+p_{i}\right)_{\mu}$ is the mean momentum. We have the constraints $G\left(q^{2}=0\right)=1$ from current conservation and $F_{1}\left(q^{2}=0\right)=1$ by energy-momentum conservation.

The contributions to gravitational Compton scattering from the seagull, direct-Born and cross-Born diagrams is then easily found and is only slightly more complex than the corresponding electromagnetic forms:

$$
\begin{aligned}
\operatorname{Amp}_{G C}^{2 a}(S=0) & =\kappa^{2}\left[\epsilon _ { f } ^ { * } \cdot \epsilon _ { i } \left(\epsilon_{i} \cdot p_{i} \epsilon_{f}^{*} \cdot p_{f}\right.\right. \\
& \left.\left.+\epsilon_{i} \cdot p_{f} \epsilon_{f}^{*} \cdot p_{i}\right)-\frac{1}{2} k_{i} \cdot k_{f}\left(\epsilon_{f}^{*} \cdot \epsilon_{i}\right)^{2}\right] \\
\operatorname{Amp}_{G C}^{2 b}(S=0) & =2 \kappa^{2} \frac{\left(\epsilon_{i} \cdot p_{i}\right)^{2}\left(\epsilon_{f}^{*} \cdot p_{f}\right)^{2}}{p_{i} \cdot k_{i}} \\
\operatorname{Amp}_{G C}^{2 c}(S=0) & =-2 \kappa^{2} \frac{\left(\epsilon_{f}^{*} \cdot p_{i}\right)^{2}\left(\epsilon_{i} \cdot p_{f}\right)^{2}}{p_{i} \cdot k_{f}}
\end{aligned}
$$

However, the sum of these three diagrams is neither complete nor gauge-invariant. We must add the contribution from Figure 2d, the graviton pole diagram

$$
\begin{aligned}
& \operatorname{Amp}_{G C}^{2 d}(S=0)=\frac{4 \kappa^{2}}{k_{i} \cdot k_{f}}\left[\epsilon_{f}^{*} \cdot p_{f} \epsilon_{f}^{*} \cdot p_{i}\left(\epsilon_{i} \cdot\left(p_{i}-p_{f}\right)\right)^{2}\right. \\
+ & \epsilon_{i} \cdot p_{i} \epsilon_{i} \cdot p_{f}\left(\epsilon_{f}^{*} \cdot\left(p_{i}+p_{f}\right)\right)^{2} \\
+ & \epsilon_{i} \cdot\left(p_{i}-p_{f}\right) \epsilon_{f}^{*} \cdot\left(p_{f}-p_{i}\right)\left(\epsilon_{f}^{*} \cdot p_{f} \epsilon_{i} \cdot p_{i}+\epsilon_{f}^{*} \cdot p_{i} \epsilon_{i} \cdot p_{f}\right) \\
- & \epsilon_{f}^{*} \cdot \epsilon_{i}\left(\epsilon_{i} \cdot\left(p_{i}-p_{f}\right) \epsilon_{f}^{*} \cdot\left(p_{f}-p_{i}\right)\left(p_{i} \cdot p_{f}-m^{2}\right)\right. \\
+ & k_{i} \cdot k_{f}\left(\epsilon_{f}^{*} \cdot p_{f} \epsilon_{i} \cdot p_{i}+\epsilon_{f}^{*} \cdot p_{i} \epsilon_{i} \cdot p_{f}\right) \\
+ & \epsilon_{i} \cdot\left(p_{i}-p_{f}\right)\left(\epsilon_{f}^{*} \cdot p_{f} p_{i} \cdot k_{f}+\epsilon_{f}^{*} \cdot p_{i} p_{f} \cdot k_{f}\right) \\
+ & \left.\epsilon_{f}^{*} \cdot\left(p_{f}-p_{i}\right)\left(\epsilon_{i} \cdot p_{i} p_{f} \cdot k_{i}+\epsilon_{i} \cdot p_{f} p_{i} \cdot k_{i}\right)\right) \\
+ & \left(\epsilon_{f}^{*} \cdot \epsilon_{i}\right)^{2}\left(p_{i} \cdot k_{i} p_{f} \cdot k_{i}+p_{i} \cdot k_{f} p_{f} \cdot k_{f}\right. \\
- & \frac{1}{2}\left(p_{i} \cdot k_{i} p_{f} \cdot k_{f}+p_{i} \cdot k_{f} p_{f} \cdot k_{i}\right) \\
+ & \left.\left.\frac{3}{2} k_{i} \cdot k_{f}\left(p_{i} \cdot p_{f}-m^{2}\right)^{2}\right)\right]
\end{aligned}
$$

where the complex form given here arises from the graviton propagator

$$
D_{\alpha \beta ; \gamma \delta}=\frac{i P_{\alpha \beta ; \gamma \delta}}{q^{2}+i \epsilon} \quad \text { with } \quad P_{\alpha \beta ; \gamma \delta}\left[I_{\alpha \beta ; \gamma \delta}-\frac{1}{2} \eta_{\alpha \beta} \eta_{\gamma \delta}\right]
$$

and the triple graviton vertex, which is a consequence of the nonlinearity of general relativity [22]

$$
\begin{aligned}
& \tau_{\alpha \beta, \gamma \delta}^{\mu \nu}(k, q)=\frac{i \kappa}{2}\left\{\left(I_{\alpha \beta, \gamma \delta}-\frac{1}{2} \eta_{\alpha \beta} \eta_{\gamma \delta}\right)\right. \\
& \times\left[k^{\mu} k^{v}+(k-q)^{\mu}(k-q)^{v}+q^{\mu} q^{v}-\frac{3}{2} \eta^{\mu v} q^{2}\right] \\
& +2 q_{\lambda} q_{\sigma}\left[I^{\lambda \sigma,}{ }_{\alpha \beta} I^{\mu \nu,}{ }_{\gamma \delta}+I^{\lambda \sigma,}{ }_{\gamma \delta} I^{\mu \nu,}{ }_{\alpha \beta}\right.
\end{aligned}
$$

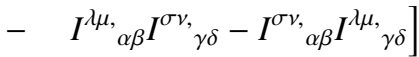

$$
\begin{aligned}
& +\left[q_{\lambda} q^{\mu}\left(\eta_{\alpha \beta} I^{\lambda v,}{ }_{\gamma \delta}+\eta_{\gamma \delta} I^{\lambda v}{ }_{\alpha \beta}\right)\right. \\
& +q_{\lambda} q^{\nu}\left(\eta_{\alpha \beta} I^{\lambda \mu}{ }_{\gamma \delta}+\eta_{\gamma \delta} I^{\lambda \mu,}{ }_{\alpha \beta}\right) \\
& \text { - } q^{2}\left(\eta_{\alpha \beta} I^{\mu \nu,}{ }_{\gamma \delta}+\eta_{\gamma \delta} I^{\mu \nu,}{ }_{\alpha \beta}\right) \\
& \text { - } \left.\eta^{\mu v} q^{\lambda} q^{\sigma}\left(\eta_{\alpha \beta} I_{\gamma \delta, \lambda \sigma}+\eta_{\gamma \delta} I_{\alpha \beta, \lambda \sigma}\right)\right] \\
& +\left[2 q ^ { \lambda } \left(I^{\sigma \nu,}{ }_{\alpha \beta} I_{\gamma \delta, \lambda \sigma}(k-q)^{\mu}+I^{\sigma \mu,}{ }_{\alpha \beta} I_{\gamma \delta, \lambda \sigma}(k-q)^{\nu}\right.\right. \\
& \text { - } \left.I^{\sigma v,}{ }_{\gamma \delta} I_{\alpha \beta, \lambda \sigma} k^{\mu}-I^{\sigma \mu \mu}{ }_{\gamma \delta} I_{\alpha \beta, \lambda \sigma} k^{\nu}\right) \\
& +q^{2}\left(I^{\sigma \mu}{ }_{\alpha \beta} I_{\gamma \delta, \sigma}{ }^{\nu}+I_{\alpha \beta, \sigma}{ }^{\nu} I^{\sigma \mu}{ }_{\gamma \delta}\right) \\
& \left.+\eta^{\mu v} q^{\lambda} q_{\sigma}\left(I_{\alpha \beta, \lambda \rho} I^{\rho \sigma,}{ }_{\gamma \delta}+I_{\gamma \delta, \lambda \rho} I^{\rho \sigma,}{ }_{\alpha \beta}\right)\right] \\
& +\left[( k ^ { 2 } + ( k - q ) ^ { 2 } ) \left(I^{\sigma \mu}{ }_{\alpha \beta} I_{\gamma \delta, \sigma}{ }^{\nu}+I^{\sigma \nu,}{ }_{\alpha \beta} I_{\gamma \delta, \sigma}{ }^{\mu}\right.\right. \\
& \text { - } \left.\frac{1}{2} \eta^{\mu v} P_{\alpha \beta, \gamma \delta}\right) \\
& \text { - } \left.\left.\quad\left(k^{2} \eta_{\gamma \delta} I^{\mu \nu,}{ }_{\alpha \beta}+(k-q)^{2} \eta_{\alpha \beta} \mu_{\gamma \delta}^{\mu \nu}\right)\right]\right\} \\
& I_{\alpha \beta ; \gamma \delta}=\frac{1}{2}\left(\eta_{\alpha \gamma} \eta_{\beta \delta}+\eta_{\alpha \delta} \eta_{\beta \gamma}\right)
\end{aligned}
$$

where

Adding the four diagrams together we find the final gaugeinvariant form. Obviously the overall structure of the result is complex and difficult to understand. However, there occurs a miraculous simplification, which goes under the name of the "double copy theorem", which asserts that the gravitational Compton amplitude can be factorized 
via [5],[6].

$\operatorname{Amp}_{C G}^{t o t}(S=0)=\sum_{i=a}^{d} \operatorname{Amp}_{G C}^{2 i}(S=0)=F \cdot\left(\operatorname{Amp}_{C}^{t o t}(S=0)\right)^{2}$

where $\operatorname{Amp}_{C}(S=0)$ is the spin-0 Compton amplitude given in Eq. (3) and

$$
F=\frac{\kappa^{2}}{8 e^{4}} \frac{p_{i} \cdot k_{i} p_{i} \cdot k_{f}}{k_{i} \cdot k_{f}}
$$

is a kinematic factor. We leave it to the reader to verify this identity. Remarkably this factorization also obtains for general spin via

$\operatorname{Amp}_{C G}^{t o t}(S)=\sum_{i=a}^{d} \operatorname{Amp}_{G C}^{2 i}(S)=F \cdot \operatorname{Amp}_{C}^{t o t}(S=0) \cdot \operatorname{Amp}_{C}^{t o t}(S)$

We can demonstrate this explicitly for the simplest case of spin- $\frac{1}{2}$ for which the general form of the energymomentum tensor matrix element is [21]

$$
\begin{aligned}
& \left\langle p_{f}\left|T_{\mu v}(x)\right| p_{i}\right\rangle=e^{-i q \cdot x} \bar{u}\left(p_{f}\right) \\
\times \quad & {\left[\left(P_{\mu} \gamma_{\nu}+P_{\nu} \gamma_{\mu}\right) F_{1}\left(q^{2}\right)\right.} \\
- & \frac{i}{2 m}\left(P_{\mu} \sigma_{\nu \lambda} q^{\lambda}+P_{\nu} \sigma_{\mu \lambda} q^{\lambda}\right) F_{2}\left(q^{2}\right) \\
+ & \left.\frac{1}{4 m^{2}}\left(q_{\mu} q_{v}-\eta_{\mu \nu} q^{2}\right) F_{3}\left(q^{2}\right)\right] u\left(p_{i}\right)
\end{aligned}
$$

where now $F_{1}\left(q^{2}=0\right)=1$ from energy-momentum conservation and $F_{2}\left(q^{2}=0\right)=1$ from angular-momentum conservation. The latter condition requires the vanishing of the anomalous gravitomagnetic moment. Using the lowest order forms, we find for gravitational Compton scattering

$$
\begin{array}{ll} 
& \left.\operatorname{Amp}_{G C}^{2 a}\left(S=\frac{1}{2}\right)\right)=\kappa^{2} \bar{u}\left(p_{f}\right) \\
\times \quad & {\left[\frac{3}{16} \epsilon_{f}^{*} \cdot \epsilon_{i}\left(\xi_{i} \epsilon_{f}^{*} \cdot\left(p_{i}+p_{f}\right)+\xi_{f}^{*} \epsilon_{i} \cdot\left(p_{i}+p_{f}\right)\right)\right.} \\
+ & \left.\quad \frac{i}{8} \epsilon_{f}^{*} \cdot \epsilon_{i} \epsilon^{\rho \sigma \eta \lambda} \gamma_{\lambda} \gamma_{5}\left(\epsilon_{i \eta} \epsilon_{f \sigma}^{*} k_{f \rho}-\epsilon_{f \eta}^{*} \epsilon_{i \sigma} k_{i \rho}\right)\right] u\left(p_{i}\right) \\
& \mathrm{Amp}_{G C}^{2 b}\left(S=\frac{1}{2}\right)=\kappa^{2} \frac{\epsilon_{f}^{*} \cdot p_{f} \epsilon_{i} \cdot p_{i}}{8 p_{i} \cdot k_{i}} \\
\times \quad & \bar{u}\left(p_{f}\right)\left[\xi_{f}^{*}\left(p_{i}+k_{i}+m\right) \xi_{i}\right] u\left(p_{i}\right) \\
& \mathrm{Amp}_{G C}^{2 c}\left(S=\frac{1}{2}\right)=-\kappa^{2} \frac{\epsilon_{f}^{*} \cdot p_{i} \epsilon_{i} \cdot p_{f}}{8 p_{i} \cdot k_{f}} \\
\times \quad & \bar{u}\left(p_{f}\right)\left[\xi_{i}\left(p_{i}-\not k_{f}+m\right) \xi_{f}^{*}\right] u\left(p_{i}\right) \\
& \mathrm{Amp}_{G C}^{2 d}\left(S=\frac{1}{2}\right)=\frac{\kappa^{2}}{k_{i} \cdot k_{f}} \bar{u}\left(p_{f}\right) \\
\times \quad & {\left[\left(\xi_{i} \epsilon_{f}^{*} \cdot k_{i}+\xi_{f}^{*} \epsilon_{i} \cdot k_{f}\right)\right.} \\
\times \quad & \left(\epsilon_{i} \cdot p_{i} \epsilon_{f}^{*} \cdot p_{f}-\epsilon_{i} \cdot p_{f} \epsilon_{f}^{*} \cdot p_{i}\right) \\
-\quad & \left(\epsilon_{f}^{*} \cdot \epsilon_{i}\right)\left(k_{i} \cdot k_{f}\left(\xi_{f}^{*} \epsilon_{i} \cdot k_{f}+\xi_{i} \epsilon_{f}^{*} \cdot p_{i}\right)\right. \\
+\quad & k_{i}\left(\epsilon_{f}^{*} \cdot p_{f} \epsilon_{i} \cdot p_{i}-\epsilon_{f}^{*} \cdot p_{i} \epsilon_{i} \cdot p_{f}\right) \\
+\quad & \left.p_{i} \cdot k_{i}\left(\xi_{i} \epsilon_{f}^{*} \cdot k_{i}+\xi_{f}^{*} \epsilon_{i} \cdot k_{f}\right)\right) \\
+\quad & \left.\left(\epsilon_{f}^{*} \cdot \epsilon_{i}\right)^{2} k_{i}\left(p_{i} \cdot k_{i}-\frac{1}{2} k_{i} \cdot k_{f}\right)\right] u\left(p_{i}\right)
\end{array}
$$

Again we leave it as an exercise to show that

$$
\begin{gathered}
\operatorname{Amp}_{C G}^{t o t}\left(S=\frac{1}{2}\right)=\sum_{i=a}^{d} \operatorname{Amp}_{G C}^{2 i}\left(S=\frac{1}{2}\right) \\
=F \cdot \operatorname{Amp}_{C}^{t o t}(S=0) \cdot \operatorname{Amp}_{C}^{t o t}\left(S=\frac{1}{2}\right)
\end{gathered}
$$

In the case of gravitational Compton scattering, Low's theorem reads [9].

$$
\operatorname{Amp}_{G C}(S)=\operatorname{Amp}_{G C}^{B o r n}(S)+\operatorname{Amp}_{G C}^{N B}(S)
$$

where $\operatorname{Amp}_{G C}^{B o r n}(S)$ is the sum of the four diagrams shown in Figure 2 and $\operatorname{Amp}_{G C}^{N B}(S)=O\left(\omega^{4}\right)$. The origin of the non-Born terms is again associated with polarizability effects but now represents a quadrupole polarizability [23]. Recall that in the electromagnetic case the polarizability $\alpha_{E}, \beta_{M}$ represents an induced dipole moment which is produced in the presence of an external electromagnetic field. In the gravitational case, the polarizability represents the induced quadrupole moment which is generated in the presence of an external gravitational field gradient

$$
Q_{i j}=\alpha_{G} R_{0 i ; 0 j}
$$

where

$$
Q_{i j}=\int d^{3} r \rho(\boldsymbol{r})\left(\frac{3}{2} r_{i} r_{j}-\frac{1}{2} \delta_{i j} \boldsymbol{r}^{2}\right)
$$

is the quadrupole moment and $R_{\alpha \beta ; \gamma \delta}$ is the curvature tensor [23]. The corresponding effective Lagrangian is

$$
\mathcal{L}_{e f f}=-\frac{1}{2} \alpha_{G} R_{\alpha \beta ; \gamma \delta} R^{\rho \beta ; \sigma \delta}\left(\partial^{\alpha} \partial^{\gamma} \phi^{\dagger}\right) \partial_{\rho} \partial_{\sigma} \phi
$$

Then just as the electromagnetic polarizability leads, via two-photon exchange, to the long range CasimirPolder interaction [24]

$$
V_{e m}(r)=-\frac{23\left(\alpha_{E}^{(1)} \alpha_{E}^{(2)}+\beta_{M}^{(1)} \beta^{(2)}\right)-7\left(\alpha_{E}^{(1)} \beta_{M}^{(2)}+\beta_{M}^{(1)} \alpha_{E}^{(2)}\right)}{4 \pi r^{7}}
$$

the gravitational polarizability leads, via two-graviton exchange, to a long-range interaction [23]

$$
V_{\text {grav }}(r)=-\frac{3897 \alpha_{G}^{(1)} \alpha_{G}^{(2)}}{1024 \pi^{3} r^{11}}
$$

Because of the parallels between the electromagnetic and gravitational interactions, it is interesting to ask if there is a gravitational analog to the GDH sum rule, which if so would have a form

$$
0=\frac{1}{2 \pi^{2}} \int_{0}^{\infty} \frac{d \omega^{\prime}\left(\sigma_{\frac{5}{2}}\left(\omega^{\prime}\right)-\sigma_{\frac{3}{2}}\left(\omega^{\prime}\right)\right)}{\omega^{\prime}}
$$

because of the vanishing of the anomalous gravitomagnetic moment noted above. A 1968 paper by Gross and Jackiw shows that fixed-angle dispersion relations do exist [9], but there is an important wrinkle which asserts that sum rules such as Eq. (48) do not. The important point here can be seen by use of factorization, which asserts that 
in the case of spinless scattering we have the forward scattering amplitude

$$
\operatorname{Amp}_{C}(S=0)(\theta) \stackrel{\theta \rightarrow 0}{\longrightarrow}-2 e^{2}
$$

We have then

$$
\operatorname{Amp}_{G C}(\theta) \stackrel{\theta \rightarrow 0}{\longrightarrow}\left(-2 e^{2}\right)^{2} \cdot \frac{\kappa^{2}}{8 e^{4}} \frac{1}{1-\cos \theta}=\frac{\kappa^{2}}{2(1-\cos \theta)}
$$

so that the forward-angle gravitational Compton amplitude becomes infinite. Because of this divergence, the optical theorem [11]

$$
\operatorname{Im} f(\theta)=\frac{\omega}{4 \pi} \sigma_{t o t}
$$

cannot be used. It is interesting to note that in the case of the Compton scattering of a particle with spin

$$
\operatorname{Amp}_{C}(S)(\theta) \stackrel{\theta=0}{\longrightarrow}-2 e^{2} \boldsymbol{\epsilon}_{i} \cdot \boldsymbol{\epsilon}_{f}^{*}+i \boldsymbol{\sigma} \cdot \boldsymbol{\epsilon}_{f}^{*} \times \boldsymbol{\epsilon}_{i} \frac{t}{2\left(s-m^{2}\right)}+\ldots
$$

We see explicitly then that the spin-flip Compton amplitude in the forward direction vanishes, so that only the anomalous magnetic moment contributes. However, in the gravitational case, because the factor $F \stackrel{t \rightarrow 0}{\longrightarrow} \sim 1 / t$ the gravitational Compton spin-flip amplitude is nonvanishing

$$
\begin{aligned}
& \operatorname{Amp}_{G C}(S)(\theta) \stackrel{\theta=0}{\longrightarrow} 8 \pi G\left[\left(\boldsymbol{\epsilon}_{i} \cdot \boldsymbol{\epsilon}_{f}^{*}\right)^{2} \frac{\left(s-m^{2}\right)^{2}}{t}\right. \\
+ & \left.i \boldsymbol{\sigma} \cdot \boldsymbol{\epsilon}_{f}^{*} \times \boldsymbol{\epsilon}_{i} \frac{s-m^{2}}{2}+\ldots\right]
\end{aligned}
$$

However, the cross section still diverges so that there exists no gravitational analog of the GDH sum rule.

\section{Gravitational Scattering}

In the previous section we showed how the factorization implied by the double copy theorem could be used to evaluate the gravitational scattering reaction in terms of relatively simple Compton scattering amplitudes. In this section we show how this result can be employed to evaluate the one loop corrections to electromagnetic or gravitational scattering. At lowest order, of course, the results of single-photon or single-graviton exchange are well-known and lead to the Coulomb and Newtonian interactions when Fourier-transformed. That is, in the case of photon exchange between systems having charge $Q_{1} e$ and $Q_{2} e$, we have, in the nonrelativistic limit,

$$
\begin{aligned}
\mathcal{M}_{e m}(q)= & \frac{Q_{1} e Q_{2} e}{\sqrt{16 E_{1} E_{2} E_{3} E_{4}}}\left(p_{1}+p_{2}\right)^{\mu} \frac{\eta_{v v}}{q^{2}}\left(p_{3}+p_{4}\right)^{v} \\
& \stackrel{N R}{\longrightarrow} \frac{Q_{1} Q_{2} e^{2}}{\boldsymbol{q}^{2}}
\end{aligned}
$$

so

$$
V_{e m}^{(0)}(r)=\int \frac{d^{3} q}{(2 \pi)^{3}} e^{-i \boldsymbol{q} \cdot \boldsymbol{r}} \mathcal{M}_{\gamma}(q)=\frac{Q_{1} Q_{2} \alpha}{|\boldsymbol{r}|}
$$

while one-graviton exchange between systems having mass $m_{1}$ and $m_{2}$ is

$$
\begin{aligned}
\mathcal{M}_{\text {grav }}(q)= & \frac{\frac{\kappa}{2} m_{1} \frac{\kappa}{2} m_{2}}{\sqrt{16 E_{1} E_{2} E_{3} E_{4}}} \frac{1}{2}\left(p_{1}+p_{2}\right)^{\mu}\left(p_{1}+p_{2}\right)^{v} \\
\times & \frac{P_{\mu v ; \alpha \beta}}{q^{2}} \frac{1}{2}\left(p_{3}+p_{4}\right)^{\alpha}\left(p_{3}+p_{4}\right)^{\beta} \\
& \stackrel{N R}{\longrightarrow}-\frac{\kappa^{2} m_{1} m_{2}}{8 \boldsymbol{q}^{2}}
\end{aligned}
$$

so

$$
V_{\text {grav }}^{(0)}(r)=\int \frac{d^{3} q}{(2 \pi)^{3}} e^{-i \boldsymbol{q} \cdot \boldsymbol{r}} \mathcal{M}_{\text {grav }}(q)=-\frac{G m_{1} m_{2}}{|\boldsymbol{r}|}
$$

These well-known classical physics results are augmented in the quantum mechanical case by loop corrections, and the one-loop corrections to these forms can be found by the use of so-called on-shell methods. In this technique, one uses unitarity to determine the discontinuity across the two-photon or two graviton t-channel cuts (cf. Figure 3) [25].

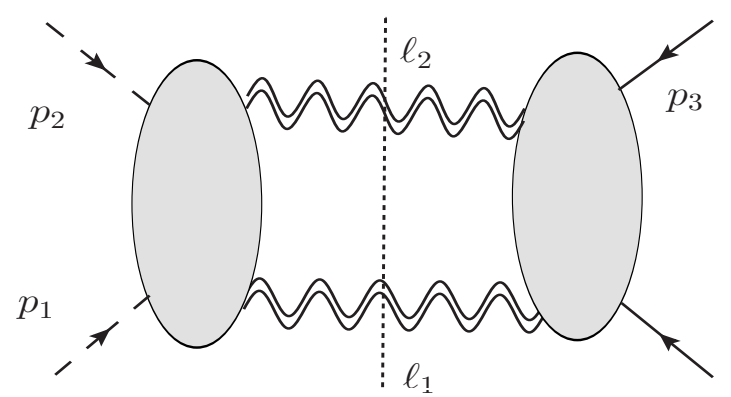

Figure 3. The two photon cut for the amplitude between a massive scalar particles. The grey blobs represent tree-level Compton amplitudes.

Thus, in the gravitational case we have the form

$$
\begin{aligned}
& \operatorname{Disc} \operatorname{Amp}_{G}\left(p_{3}, p_{4} ; p_{1}, p_{2}\right)(S) \\
& =\frac{-i}{2 !} \frac{1}{\sqrt{16 E_{1} E_{2} E_{3} E_{4}}} \int \frac{d^{3} \ell_{1}}{2 \ell_{1}^{0}(2 \pi)^{3}} \int \frac{d^{3} \ell_{2}}{2 \ell_{2}^{0}(2 \pi)^{3}} \\
& \times(2 \pi)^{4} \delta^{4}\left(p_{1}+p_{2}-\ell_{1}-\ell_{2}\right) \operatorname{Amp}_{G C}^{* \rho \sigma ; \xi \zeta}\left(p_{3} p_{4} ; \ell_{1} \ell_{2}\right)(S) \\
& \times \quad P_{\rho \sigma ; \alpha \beta} P_{\xi \zeta ; \gamma \delta} \operatorname{Amp}_{G C}^{\alpha \beta, \gamma \delta}\left(\ell_{1} \ell_{2} ; p_{1} p_{2}\right)(S)
\end{aligned}
$$

Using factorization, this discontinuity calculation can be written in terms of simple Compton amplitudes. In this way the discontinuity of one-loop corrections to the gravitational scattering of spinless systems can be straightforwardly found to be

$$
\begin{array}{r}
\operatorname{Disc~Amp}_{G}\left(p_{3} p_{4} ; p_{1} p_{2}\right)(S=0)=-i \frac{\kappa^{4} m_{A} m_{B}}{128 \pi} \\
\times\left[\frac{6 \pi\left(m_{A}+m_{B}\right)}{\sqrt{-q^{2}}}+\frac{41}{5}+4 \pi i \frac{m_{A} m_{B} m_{r}}{p_{0} q^{2}}+\ldots\right]
\end{array}
$$


The scattering amplitude itself then is

$$
\begin{aligned}
& \operatorname{Amp}_{G}\left(p_{3} p_{4} ; p_{1} p_{2}\right)(S=0)=-\frac{\kappa^{4} m_{A} m_{B}}{1024 \pi^{2}} \\
\times & {\left[-\frac{6 \pi\left(m_{A}+m_{B}\right)}{\sqrt{-q^{2}}}+\frac{41}{5} \log -q^{2}\right.} \\
+ & \left.4 \pi i \frac{m_{A} m_{B} m_{r}}{p_{0} q^{2}} \log -q^{2}+\ldots\right]
\end{aligned}
$$

where $m_{r}=m_{A} m_{B} /\left(m_{A}+m_{B}\right)$ is the reduced mass and $p_{0}$ is the magnitude of the center of mass frame momentum. Eq. (60) agrees with the result calculated by Bjerrum-Bohr et al. and by Kirilin and Khriplovich by much more laborious Feynman diagram methods [27],[26]. The imaginary piece of the scattering amplitude arises from the second Born approximation, which is subtracted when defining the second order potential

$$
\begin{aligned}
V_{\text {grav }}^{(1)}(r) & =\int \frac{d^{3} q}{(2 \pi)^{3}} e^{-i \boldsymbol{q} \cdot \boldsymbol{r}}\left(\mathcal{M}_{\text {grav }}(q)-\operatorname{Amp}_{\text {Born }}^{(2)}(q)\right) \\
& =-\frac{3 G^{2} m_{A} m_{B}\left(m_{A}+m_{B}\right)}{r^{2}}-\frac{41 G^{2} m_{A} m_{B} \hbar}{10 \pi r^{3}}
\end{aligned}
$$

The simplification here comes from a reordering of summation and integration. That is, in the conventional procedure one evaluates individual and gauge-dependent Feynman diagrams (including ghost loops) by performing fourdimensional integrations. The various diagrams are then summed to produce the final gauge-invariant result. In the on-shell procedure the summation is performed first, producing gauge-invariant Compton amplitudes, which are then used as a component of a two-dimensional integration. Also, because the amplitudes are on-shell, no ghost contributions are required. In this way what was originally a two-year calculation becomes a two-day evaluation.

\section{Conclusions}

Above we have discussed how Compton scattering- $S+$ $\gamma \rightarrow S+\gamma$-has been used to probe the structure of a system with spin-S by measurement of its response when placed in external electromagnetic fields. The resulting deformations are described in terms of polarizabilities [2], which in the case of the proton have been the subject of experimental study for many years at various laboratories [7]. Most recently experiments by the A2 collaboration at MAMI utilizing polarization have measured not only the size of the leading electric and magnetic polarizabilities $\alpha_{E}$ and $\beta_{M}$ but also of the higher order spin polarizabilities $\gamma_{E 1 E 1}, \gamma_{M 1 M 1}, \gamma_{E 2 M 1}$, and $\gamma_{M 2 E 1}$ [20]. On the theoretical side these polarizabilities have been calculated via various methods, most recently by the means of chiral perturbation theory [17],[18]. The chipt estimates are in basic agreement with the experimental results, which indicates the importance of the pion cloud in studying such quantities.
The recent LIGO measurement of gravitational waves and the confirmation thereby of the existence of gravitons [1] emphasizes the need to study the parallel gravitaional Compton scattering reaction- $S+g \rightarrow S+g-$ and this reaction was discussed in turn. The standard way to calculate such reactions, by the use of Feynman diagrams, was shown to be enormously simplified by the use of te recently discovered double-copy theorem [5], which asserts that the desired gravitational Compton amplitudes can be written in terms of a product of ordinary Compton amplitudes together with a simple kinematic factor. This procedure was then shown to allow, via the use of unitarity and on-shell methods, a relatively simple evaluation of the one-loop corrections to gravitational scattering by evaluation of the discontinuity over the two-graviton t-channel cut.

\section{Acknowledgement}

It is a pleasure to acknowledge the warm hospitality of the Bonn physics group.

\section{References}

[1] B.P. Abbott et al., Phys. Rev. Lett. 116, 061102 (2016).

[2] B.R. Holstein and S. Scherer, Ann. Rev. Nucl. Part. Sci. 64, 51-81 (2014).

[3] S. Gerasimov, Yad. Fiz. 2, 598 (1965).

[4] S.D. Drell and A.C. Hearn, Phys. Rev. Lett. 16, 908 (1966).

[5] See, e.g., Z. Bern, Living Rev. Relativity 5, 5 (2002).

[6] S.Y. Choi et al., Phys. Rev. D51, 2751 (1995).

[7] See, e.g. F. Hagelstein et al., Prog. Part. Nucl. Phys. 88, 29 (2016).

[8] F.E. Low, Phys. Rev. 96, 1428 (1954).

[9] D.J. Gross and R. Jackiw, Phys. Rev. 166, 1287 (1968).

[10] K.A. Olive et al., Chin. Phys. C38, 090001 (2014).

[11] See, e.g., E. Merzbacher, Quantum Mechanics, Wiley, New York (1972).

[12] N.C. Mukhopadhyay et al., Phys. Rev. D47, (1993).

[13] D. Drechsel et al., Phys. Rept. 378, 99 (2003).

[14] A.M. Baldin, Nucl. Phys. 18, 310 (1960); L.I. Lapidus, Sov. Phys. JETP 16, 964 (1963).

[15] B.R. Holstein et al., Phys. Rev. C61, 034316 (2000).

[16] J. Gasser, M.E. Sainio, and A. Svarc, Nucl. Phys. B307 779 (1988).

[17] V. Bernard, N. Kaiser, and U.-G. Meißner, Int. J. Mod. Phys. E193 (1995).

[18] V. Lensky and V. Pascalutsa, Eur. Phys. J. C65, 195 (2010).

[19] B. Pasquini, D. Drechsel, and M. Vanderhaeghen, Phys. Rev. C76, 015203 (2007).

[20] P.P. Martel et al., Phys. Rev. Lett. 114, 112501 (2015).

[21] See e.g., J.F. Donoghue and B.R. Holstein, J. Phys. G42, 103102 (2015). 
[22] J.F. Donoghue, Phys. Rev. D50, 3874 (1994).

[23] L.H. Ford et al., Phys. Rev. Lett. 116151301 (2016).

[24] H.B.G. Casimir and D. Polder, Phys. Rev. 73, 366 (1948).

[25] N.E.J. Bjerrum-Bohr, J.F. Donoghue, and P. Vanhove, JHEP 01, 111 (2014).
[26] I.B. Khriplovich and G.G. Kirilin, Econf C0306234, 1361 (2003).

[27] N.E.J. Bjerrum-Bohr, J.F. Donoghue, and B.R. Holstein, Phys. Rev. D67, 084033 (2003). 